ring to the management of traumatic dental injuries and tooth resorption. These sections deliver evidence-based guidance (including guidance from the Royal College of Surgeons of England), and describes operative techniques for clinical management of traumatic dental injuries in superb detail so that these can be followed easily from the text.

The final section entitled 'Syndromes' discusses common syndromes associated with dental anomalies and is divided into autosomal dominant syndromes, autosomal recessive syndromes, X-linked syndromes and chromosomal syndromes.

This is a comprehensive, up-to-date guide to dental hard tissue pathology that is relevant to dental practitioners and dental students alike. Whether you are working in practice or as a hospital specialist, this book will provide you with an extensive understanding of dental hard tissue pathology as well as the current recommendations for prevention and practical management of these conditions.

S. Marshall

\section{ENDODONTIC RADIOLOGY, 2ND EDITION}

\begin{tabular}{ll}
\hline Endodontic & $\begin{array}{l}\text { B. Basrani (ed) } \\
\text { Wiley-Blackwell } \\
\text { price £93.99; pp } 448 \\
\text { ISBN } 9780470958490\end{array}$ \\
Radiology & \\
\hline &
\end{tabular}

A well-written and comprehensive text with contributions from a large number of international experts in the field of endodontics. The book delves into all aspects of radiographic imaging related to endodontics to provide a text that is current for the modern-day clinician.

The book is divided into six parts. These are further subdivided into smaller, more specific chapters. Each chapter is approximately 15-20 pages long, making it a suitable read at lunch times and generally more manageable for the busy modernday practitioner.

This book is ideal for general dental practitioners who are seeking a greater understanding in the field of endodontic radiology. It would also be highly suitable for the clinician who has committed their line of work to endodontics. The text has a great emphasis on cone beam computed tomography (CBCT), but due to the lack of teaching of this subject in undergraduate studies, trainee dentists are advised to 'proceed with caution'.

The book opens by giving the reader a sound understanding of radiology and its relevance to endodontics. Further in, the chapters go on to discuss each endodontic disease process histopathologically. This aids the reader to better understand and interpret lesions when presented on a radiograph. The final parts of the book deal with advanced imaging modalities with references to various clinical cases that bring together all the theory taught in the previous chapters. The author has done a great job at educating the reader to the limitations of plain film radiography by giving emphasis to CBCT and demonstrating its ability to provide an added dimension to visual imaging in endodontics. There are also chapters dedicated to radiographic imaging in implant dentistry and a section devoted to teaching and research; this demonstrates the sheer scope of this book.

It is my impression that all of the images in the book, of which there are plenty, are clinically relevant in aiding the reader in understanding endodontics to a much deeper and satisfying level. These range from histological slides of periapical tissues, clinical photographs and radiographic images from various imaging modalities.

Being a foundation dentist, I can profess that I have extensively expanded my knowledge base from this read. It has subsequently influenced my clinical practice and I'd strongly urge anyone who is truly committed to learning and understanding endodontic imaging to a superior level to invest their time into reading this text.

J. Malik

\section{INTEGRATED CLINICAL ORTHODONTICS}

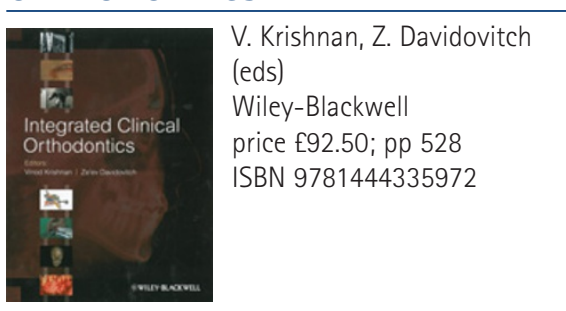

This 528-page, hard-cover book is compiled by a range of international experts. The book is divided into 25 chapters and is well-indexed throughout. Each chapter begins with a helpful summary describing the aims and objectives of the chapter.

The authors focus on the holistic approach of managing the orthodontic patient. It is stated in the preface that "orthodontics is not merely an exercise in wire bending but rather a specialty leaning on many others' and the book adheres to this philosophy throughout.

This very thorough book emphasises the interaction between orthodontics and other dental and medical specialties including periodontics, endodontics and restorative dentistry as well as prosthodontics and oral and maxillofacial surgery. In addition, congenital orofacial deformities, sleep disorders, otorhinolaryngologic, paediatric and temporomandibular joint problems are also covered.

An unusual and interesting aspect of this book is the depth of information provided on working with endocrinologists, ear, nose and throat specialists as well as orthopaedists engaged in basic research, making the book a valuable resource for hospital-based orthodontists.

The case-based discussions supplemented with clinical photographs and radiographs illustrated in this book describe patients who are medically-compromised and challenging to treat. The authors have also demonstrated this with well-illustrated cases that have been both successful and unsuccessful. Schematic diagrams and flowcharts are also widely used.

I feel that this book is well-written and easy to follow. The authors have clearly fulfilled their aims and objectives and this book will be a valuable resource for every orthodontist and anyone with a special interest in orthodontics, especially when faced with challenging clinical situations.

F. Chan 